\title{
ASPECTS OF THE THEORETICAL PERFORMANCE OF MODAL
}

\section{ADAPTIVE OPTICS}

\author{
R.W.WILSON and C.R.JENKINS \\ Royal Greenwich Observatory \\ Madingley Road \\ Cambridge \\ England \\ CBS OEZ
}

\begin{abstract}
This paper describes early results of a theoretical investigation of the performance of an adaptive optics system in which a limited number of Karhunen-Loeve modes of the wavefront distortion are corrected. We have determined the on-axis point spread function for an idealised adaptive optics system in Kolmogorov seeing, whose performance is defined in terms of radii of included energy and Strehl ratios for a range of examples. We discuss extensions of the analysis for the off-axis point spread function, and for non-Kolmogorov seeing.
\end{abstract}

\section{Introduction}

In modal adaptive optics, corrections are applied to a finite number of modes of the expansion of the wavefront distortion as a set of basis functions. A commonly chosen basis are the well known Zernike polynomials (Noll, 1976). The effects of correcting a finite number of Zernike modes have been studied by Roddier (1991), and Nakajima (1992), using analytic methods and numerical simulations. The optimal basis for a specified instrumental configuration (for which the variance of the wavefront phase after correction of a given number of modes is less than for any other possible basis) is given by the Karhunen-Loeve (KL) expansion, in which the wavefront is decomposed into an orthonormal basis with statistically independent coefficients (Fried 1978, Wang and Markey 1978). Wang and Markey showed how to calculate the PSF using a KL basis. Here we describe our implementation of these calculations and show how astronomically interesting parameters of the PSF can be deduced, and indicate the extension to more interesting cases.

\section{On-Axis Point Spread Function}

Let the complex amplitude of the wavefront at our telescope pupil, after correction of $\mathrm{N}$ KL modes be $\boldsymbol{\Phi}_{c}(\underline{r})=e^{i \phi(\underline{r})}$ inside the aperture and zero elsewhere, where $\phi(\underline{r})$ is the corrected phase distribution. In the image plane, the amplitude is $A(\underline{x})=\mathbf{F}\left[e^{i \phi(\underline{r})}\right]$ where $\mathbf{F}[]$ denotes a Fourier transform. Using the convolution theorem, the average intensity in the image plane is:

$$
\begin{aligned}
\langle I(\underline{x})\rangle & =\left\langle A(\underline{x}) A(\underline{x})^{*}\right\rangle=\left\langle\mathbf{F}\left[e x p^{i \phi(\underline{r})} \oplus e^{-i \phi(\underline{r})}\right]\right\rangle \\
& =\mathbf{F}\left[\int_{\text {aperture }}\left\langle\exp \left\{i\left[\phi\left(\underline{r}^{\prime}\right)-\phi\left(\underline{r}^{\prime}-\underline{r}\right)\right]\right\} \underline{d} r\right\rangle\right]
\end{aligned}
$$

where $\oplus$ is the convolution. From the definition of the KL functions as a complete basis set, we can write the wavefront after correction of $\mathrm{N}$ modes as $\phi(\underline{r})=$ 
$\sum_{\mathrm{n}=\mathrm{N}}^{\infty} a_{\mathrm{n}} K_{\mathrm{n}}(\underline{r})$, where $K_{\mathrm{n}}$ is the $\mathrm{n}$ 'th $\mathrm{KL}$ mode, and $a_{\mathrm{n}}$ is the corresponding expansion coefficient. By construction the $a_{n}$ are uncorrelated. Assuming Gaussian statistics, it follows that they are also independent. The average then reduces to a set of simple one parameter averages for each $a_{n}$ separately: Hence:

$$
\begin{aligned}
\langle I\rangle & =\mathbf{F}\left[\int_{\text {aperture }}\left\langle\exp \left\{i\left[\sum_{\mathrm{n}=\mathrm{N}}^{\infty} a_{\mathrm{n}}\left(K_{\mathrm{n}}\left(\underline{r}^{\prime}\right)-K_{\mathrm{n}}\left(\underline{\boldsymbol{r}}^{\prime}-\underline{r}\right)\right)\right]\right\}\right\rangle \underline{d r}\right] \\
& =\mathrm{F}\left[\int_{\text {aperture }} \exp \left\{-\frac{1}{2}\left[\sum_{\mathrm{n}=\mathrm{N}}^{\infty}\left\langle a_{\mathrm{n}}^{2}\right\rangle\left(K_{\mathrm{n}}\left(\underline{r}^{\prime}\right)-K_{\mathrm{n}}\left(\underline{r}^{\prime}-\underline{r}\right)\right]^{2}\right\} \underline{d r}\right]\right.
\end{aligned}
$$

Hence the point spread function is found numerically by evaluating the integral at each point within the aperture, and then performing a discrete Fourier transform. The infinite upper limit of the sum is replaced some finite mode number, such that the phase variance contributed by higher order modes is negligible. We have studied point spread functions for modal correction of a circular aperture for a range of values of the ratio of the aperture diameter to Fried's parameter $r_{0}$. As the number of modes removed is increased we see in each case the formation of a sharp diffraction limited core in the image, surrounded by a halo with diameter roughly equivalent to the width of the uncorrected PSF (this general result is well known).

Our main interest in these PSF's is to quantify the astronomical usefulness of partial correction by adaptive optics. Relevant quantities include photometric performance, possibilities for further deconvolution, and the implied reduction of the entrance aperture of spectrometers. We are therefore interested in the degree of energy concentration and the extent of the halo of the PSF. Figure 1 is a plot of the energy included inside circles of given radii for point spread functions in the case $\mathrm{D} / \mathrm{r}_{0}=20$, and shows how the fraction of energy inside the diffraction limited core increases with $\mathrm{N}$, with significant gains for low order correction. An often-used measure of image quality is the Strehl ratio - the ratio of the intensity of the PSF on-axis, to the diffraction limited case. When $\mathrm{N}$ is large, so that the variance $\left(\sigma^{2}\right)$ of the remaining phase distortion is much less than $2 \pi$ radians, the value of the Strehl ratio is approximated by $e^{-\sigma^{2}}$ (Tyson 1991). The value of $\sigma^{2}$ is given by the sum of the eigen values of the KL integral equation. In each case we find that the measured Strehl ratio is higher than the simple theoretical value, showing that this often quoted estimate of the expected Strehl ratio is pessimistic.

\section{Extensions of the Analysis}

Our objective in this work is to employ it as part of an idealised model of adaptive optics systems, which we intend to use for evaluating various options for the UK's programme for adaptive optics. We are trying to be both realistic but not too specific in our assumptions, particularly about the wavefront sensor and adaptive mirror. However we will be including at least the following important effects: (i) noise on the estimate of the wavefront, (ii) finite spatial and temporal resolution of the wavefront, (iii) finite bandwidth of the control system. These can be included 
quite easily in the present analysis: Noise equates to imperfect mode subtraction, resolution to an alteration of the basis set used, and bandwidth to anisoplanatic effects. A major concern for astronomical applications of adaptive optics is that the partially corrected PSF will vary over the field. We are currently engaged in calculating these off-axis PSF's. The principle is simple; the off-axis wavefront expansion is:

$$
\sum_{n=1}^{N-1}\left(a_{n}-a_{n}^{\prime}\right) K_{n}(\underline{r})+\sum_{n=N}^{\infty}\left(a_{n}\right) K_{n}(\underline{r})
$$

where the $a_{n}^{\prime}$ are the coefficients for the on-axis expansion. However a complication arises because $0 \leq\left\langle a_{n} a_{n}^{\prime}\right\rangle \leq 1$. Chassat (1989) has shown how these correlation coefficients may be calculated. The average in equation 2 then does not reduce to a set of independent univariate expectations; rather a multivariate expectation has to be calculated. It is straightforward but tedious to do this analytically, and we are engaged in a solution using a computer algebra package.

\section{References}

Chassat, F.: 1989, J. Optics (Paris) 20, 13

Fried, D.L.: 1978, J. Opt. Soc. Am. 68, 1651

Nakajima, T., Haniff, C.A.: 1992, , in preparation

Noll, R.J.: 1976, J. Opt. Soc. Am 66, 207

Roddier, F., Northcott, M., Graves, J.E., : 1991, Publs. Astron. Soc. Pacific 103, 131

Roddier, N.: 1990, Opt. Eng. 29, 1174

Tyson, R.K.: 1991, Principles of Adaptive Optics, Academic press inc.: London, 7

Wang, J.Y., Markey, J.K.: 1978, J. Opt. Soc. Am. 68, 78

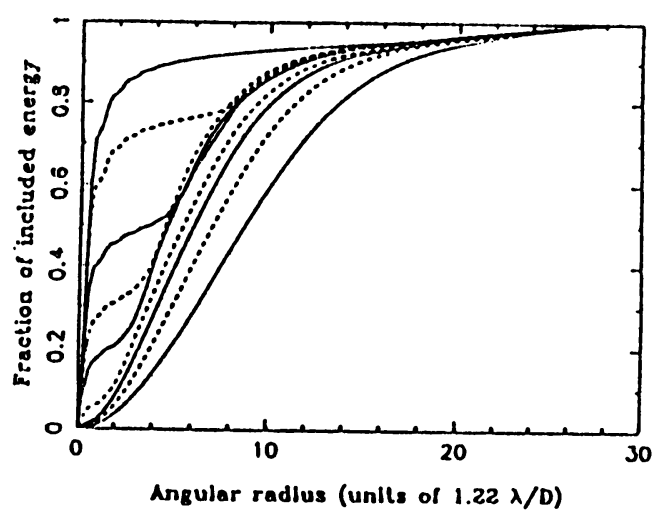

Figure 1: Radii of included energy for modal correction in the case $D / r_{0}=20$. In each case the plots are for $N=0$ (lower curve), $1,5,10,20,30,50,100$, and $\infty$ (upper curve - diffraction limited case). 